\title{
Antologías del cuento dominicano de la última década (2000-2010) y canon ${ }^{1}$
}

\author{
Rita De Maeseneer \\ University of Antwerp \\ Ilse Logie \\ University of Ghent
}

En el mundo hispánico se suele subestimar la labor no directamente ligada a los textos originales: la redacción de entradas para enciclopedias y diccionarios, prefacios, postfacios, solapas, reseñas, dirigir colecciones, hacer traducciones y organizar antologías. Todas ellas son consideradas formas subordinadas de actividad intelectual, menos nobles que la de escribir libros de teoría y crítica y, por supuesto, que la de escribir obras de creación. Sin embargo, son herramientas fundamentales en la configuración del canon cultural como lo demostró alguien como Borges, cuya Antología de la literatura fantástica (hecha en colaboración con Bioy Casares) orientó al público argentino en una dirección estética determinada: la importación de un género que los propios compiladores deseaban practicar en lengua española. En este artículo nos interesa precisamente indagar en la pertinencia de las antologías respecto a la formación del canon. Empezaremos especificando la manera como entendemos los conceptos clave: antología y canon. Luego pasaremos a nuestro estudio de caso analizando algunas antologías de cuentos dominicanos.

Aunque no es fácil encontrar una definición satisfactoria de lo que es una antología, una aproximación tentativa podría ser que una antología es un conjunto de textos y/o fragmentos de textos que se agrupan a partir de ciertas características determinadas por un seleccionador, aunque no siempre argumentadas por el mismo, y cuya finalidad principal es divulgar las obras juzgadas representativas de un autor, un género, un tema, una tendencia, un movimiento, una región o una generación. Idealmente, el antologador no es un mero coleccionista de textos, sino quien lee, desde una posición crítica, una serie de textos, que son elegidos entre lo diverso para alojarlos en una forma nueva, la antología, en cuyo seno se encuentra una sucesión de textos que interactúan entre sí y con otros textos, de acuerdo con la definición ya clásica de Claudio Guillén: "La antología es una forma colectiva intratextual que supone la reescritura o reelaboración, por parte de un lector, de textos ya existentes mediante su inserción en conjuntos nuevos" (375). Pero en la práctica, 
esta meta de cierta coherencia intratextual raras veces se cumple en las antologías que no estén centradas en una corriente literaria o una temática.

En cuanto al canon, ha corrido mucha tinta sobre este concepto sumamente resbaladizo e interpretado desde varios ángulos. Con el fin de abarcar las interpretaciones más tratadas del canon, recurriremos a las tres acepciones interrelacionadas que propone Waldo Pérez Cino en su categorización: Canon con mayúscula, canon crítico y corpus. El Canon con mayúscula es la interpretación más común, tal como fue manejada por ejemplo por Bloom en The Western Canon. Es un repertorio relativamente fijo de textos y autores cuya autoridad se considera probada y modélica y cuya influencia cultural se da por sentada. En cuanto al canon crítico, se refiere al papel de las instituciones y las academias que influyen en los raseros valorativos, expectativas retóricas o ideológicas, las metodologías o intereses que conforman el ejercicio crítico. Kermode, Guillory y Pozuelo Yvancos insistieron en esta acepción en sus acercamientos al canon. Una tercera vía para abordar el tema es mediante el estudio de un conjunto de obras y autores en un momento y en un ámbito dados, lo cual es designado por Pérez Cino con el término de corpus. Por tanto, se tratará de ver de qué manera las antologías interfieren en el canon en esas diferentes acepciones. Además, esto nos llevará inevitablemente a reflexionar sobre el papel de las antologías en relación con el paradigma de 'literatura mundial'. Investigadores como Damrosch, Moretti y Casanova han reintroducido en la crítica contemporánea esta noción. Destacan que la globalización ha complicado la idea de una 'literatura del mundo': más que para una (inalcanzable) suma de todas las literaturas o un canon unificado, reservan el término para una construcción, una especie de red inestable caracterizada por una articulación fuerte de lo local con lo universal (la que Damrosch llama la refracción elíptica). Los tres estudiosos reconocen las desigualdades y las relaciones jerárquicas que reinan en esta ágora transnacional: a pesar de la apariencia de pacifismo multicultural, se trata de una lucha constante y desigual. En este contexto, nos interesa comprobar más particularmente la aplicabilidad de algunos planteamientos de Pascale Casanova. La investigadora propone una estructura dualista del espacio literario mundial. Por un lado, hay un polo autónomo, ocupado por las literaturas asentadas en los centros y dotadas con capital simbólico. Por el otro, hay espacios literarios 'en formación' (como sería el caso dominicano), que dependen de los centros de consagración internacional. A esto se añade la heteronomía frente al absorbente poder político-nacional del propio espacio (o sea, una fuerte instrumentalización de la literatura que suele dar lugar a una estética realista). Para poder entrar en el mercado literario internacional hace falta establecer una independencia nacional y constituir un espacio literario nacional. Además, se impone un segundo paso de emancipación, que consiste en una despolitización de la literatura periférica. Frente a los escritores 'nacionales' Casanova distingue a los 'revolucionarios' o 'internacionales' cuya carrera literaria se caracteriza por dichos procesos de descolonización y desnacionalización. Para lograr tal objetivo, los revolucionarios aplican estrategias como la importación y tradición de literaturas internacionales en contra de una estética dominante (pensemos en Borges). De este modo se constata que la esfera internacional y la nacional son interdependientes: la gran fractura que explica las formas literarias y los tipos de innovación es aquélla que 
opone, en cada espacio nacional, los escritores nacionales a los internacionales. Aunque el paralelismo que Casanova establece entre internacionalidad/autonomía y regionalidad/ heteronomía es bastante mecánico, y sus argumentos no siempre se ajustan a la realidad latinoamericana por ejemplo respecto al papel de París como meridiano cultural (Sánchez Prado), su reflexión ofrece material para reflexionar. Teniendo en mente esas premisas, pasemos ahora al estudio de caso, las antologías dominicanas publicadas en la primera década del siglo XXI.

No sorprenderá que la mayor parte de las publicaciones encontradas en nuestro rastreo sean antologías de cuentos. Este género, al igual que la poesía, suele ser considerado una de las formas más idóneas a ser antologadas (Balderston ix). Para delimitar aún más el conjunto se combina a menudo con un criterio temático o de gender. Respecto a este último enfoque, dos áreas muy exitosas (y no sólo en la República Dominicana) son las antologías de mujeres, por ejemplo, Antología de cuentistas dominicanas al cuidado de Emelda Ramos en 2007, y las antologías de temática homosexual o queer, por ejemplo, Antología de la literatura gay en la República Dominicana compilada por Miguel de Camps Jiménez y Mélida García en 2004. ${ }^{2}$ Parecen sugerir que aún no se ha dado a estos dos grupos un lugar suficiente en la República de las Letras Dominicanas. A diferencia de lo que pasa en Estados Unidos, donde se está fomentando lo afro-hispano y lo multicultural, significativamente no hemos encontrado antologías de cuentos que se hayan construido en base a este criterio. A pesar de que casi cada dominicano tiene 'el negro tras la oreja', la cuestión racial (en el sentido biológico y social/multicultural) es sumamente delicada en esta media isla, donde lo negro sigue siendo asociado al haitiano frente al dominicano (Torres-Saillant). Otro criterio manejado con frecuencia es el alcance geográfico de las antologías. Por un lado, se han publicado antologías regionales, por ejemplo, El cuento contemporáneo de Santiago (1980-2005), una selección de cuentos por Máximo Vega de autores nacidos en Santiago de los Caballeros. Por otro lado, es cada vez más obvio que es imposible reducir la cuentística dominicana a los escritores residentes en la media isla. Por eso, algunas antologías dominicanas tienen en cuenta o se dedican a textos de la diáspora escritos en español y/o en inglés, con o sin traducción. Viajeros del rocío. 25 narradores de la diáspora (2008) es una publicación de la Editora Nacional de la República Dominicana al cuidado del dominicano residente en Nueva York, Rubén Sánchez Féliz. Incluye textos en español de dominicanos residentes en Estados Unidos como Franklin Gutiérrez o Rey Andújar, al igual que traducciones de dominicano-americanos como Junot Díaz. El editor distingue a tres grupos: los que se criaron y formaron en el extranjero y escriben en inglés (Junot Díaz), los que al emigrar ya tenían una obra importante en la isla (Franklin Gutiérrez, René Rodríguez Soriano) y los que emigraron jóvenes y siguen escribiendo en español, como José Carvajal (Sánchez Féliz 13). En dos de las antologías que forman parte de nuestro análisis ulterior, encontramos el mismo deseo de inclusión. Stanley advierte que Julia Álvarez y Junot Díaz no han sido antologados por "compromisos contractuales" (20). Mena señala asimismo que hubiera querido agregar a los dominicano-americanos Julia Álvarez o a Junot Díaz, pero que los excluyó por limitaciones editoriales. 
A este respecto hay que subrayar que a diferencia de Cuba y Puerto Rico las autoridades dominicanas son menos reacias a abrirse hacia la literatura en inglés escrita por autores de origen dominicano: acogen en su literatura a Junot Díaz o a Julia Álvarez (De Maeseneer). Esta actitud brinda más oportunidades a los escritores de origen dominicano de ser tomados en cuenta, ya que para su literatura no sólo Madrid o Barcelona son los meridianos culturales, sino que otro centro de gravitación es Nueva York. Efectivamente, si los críticos se interesan por la narrativa 'dominicana' es a veces más en su vertiente de literatura escrita en inglés. Así Danny Méndez publicó Narratives of Migration and Displacement in Dominican Literature, estudiando a Pedro Henríquez Ureña, y también a Junot Díaz, Josefina Báez y Loida Maritza Pérez, tres autores que escriben en un inglés salpicado en mayor o menor medida de dominicanismos. Sobre todo es intrigante el caso de Junot Díaz. Es el único escritor 'dominicano' en ser integrado en la lista de Bogotá 39 (39 autores latinoamericanos menores de 39 años), y junto con Julia Álvarez representa la República Dominicana en $A$ Whistler in the Nightworld. Short Fiction from the Latin Americas (2002) de Thomas Colchie.

Enfrentadas a este panorama, hemos optado por seleccionar cuatro antologías publicadas después de 2000 que contienen cuentos de escritores nacidos después de 1960 y que escriben español. Nos hemos propuesto estudiar qué autores contemporáneos han sido recogidos y analizar las modalidades de producción teniendo en cuenta al antologador y sus objetivos, la distribución y la recepción. De manera más concreta, hemos analizado tres antologías diacrónicas posteriores al 2000 (una de 2006 y dos de 2010), de modo que presentan asimismo una vertiente sincrónica. Van dedicadas exclusivamente a la República Dominicana y han sido elaboradas por dominicanos: Pedro Antonio Valdez, Avelino Stanley, Miguel Mena. ${ }^{3}$ A título de comparación hemos intentado buscar una antología parecida compuesta por un no dominicano y publicada en Espańa. Que sepamos no existe una antología de cuentos dominicanos más reciente que la diacrónica del 2002 al cuidado del italiano Danilo Manera para Siruela. El crítico presentó el trabajo de ocho cuentistas con tres cuentos cada uno, la mayoría de ellos ya consagrados y nacidos antes de $1960 .{ }^{4}$ Por eso hemos recurrido al cuarto volumen de Pequeñas Resistencias, una antología sincrónica de 2005, dedicada al "nuevo cuento norteamericano y caribeño". 5 Incluye Estados Unidos, México, Cuba, la República Dominicana, Puerto Rico. A diferencia de los otros volúmenes de Pequeñas Resistencias donde para cada país se escogió a un antólogo del país de origen, para el Caribe hispano y para América del norte se encomendó la tarea a dos cubanos. Para las tres islas caribeñas Ronaldo Menéndez, un cubano residente en España desde 2004, seleccionó a seis cubanos, tres dominicanos y tres puertorriqueños. ${ }^{6}$ Desde Europa/España y Estados Unidos, Cuba es la isla caribeña privilegiada: lo que se lee y lo que se traduce más actualmente del Caribe hispano es la literatura cubana, es la que más importa en el 'corpus'. La literatura dominicana sería la periferia de la periferia caribeña, si aplicáramos planteamientos hegemónicos. Reproducimos los datos relevantes para las cuatro antologías estudiadas e indicamos la cantidad de autores nacidos después de 1960 (> 1960): 


\begin{tabular}{|c|c|c|c|c|}
\hline Antologador & Año & Título & Editorial & Varios \\
\hline $\begin{array}{l}\text { Menéndez, } \\
\text { Ronaldo } \\
\text { (cubano residente } \\
\text { en ESP) }\end{array}$ & 2005 & $\begin{array}{l}\text { Pequeñas Resistencias/4. } \\
\text { Antología del nuevo cuento } \\
\text { norteamericano y caribeño }\end{array}$ & $\begin{array}{l}\text { Páginas de } \\
\text { Espuma } \\
\text { (Madrid) }\end{array}$ & $\begin{array}{l}\text { Sincrónica. } \\
3>1960\end{array}$ \\
\hline $\begin{array}{l}\text { Mena, Miguel } \\
\text { (Dominicano } \\
\text { residente en } \\
\text { Berlín) }\end{array}$ & 2010 & $\begin{array}{l}\text { Cuentos dominicanos. } \\
\text { Antología }\end{array}$ & $\begin{array}{l}\text { Cielonaranja } \\
\text { (Berlín) }\end{array}$ & $\begin{array}{l}\text { Diacrónica+sincrónica. } \\
10 / 40>1960\end{array}$ \\
\hline $\begin{array}{l}\text { Stanley, Avelino } \\
\text { (RD) }\end{array}$ & 2010 & $\begin{array}{l}\text { Ruptura del limite. Cuentos } \\
\text { dominicanos }\end{array}$ & $\begin{array}{l}\text { Cangrejo } \\
\text { Editores } \\
\text { (Bogotá) }\end{array}$ & $\begin{array}{l}\text { Diacrónica } \\
\text { generación del sesenta: } \\
\text { 9/generación de los } 80 \text { : } \\
\text { 3/19>1960 } \\
\text { Sincrónica: generación de la } \\
\text { internet: } 4>1960\end{array}$ \\
\hline $\begin{array}{l}\text { Valdez, Pedro } \\
\text { Antonio (RD) }\end{array}$ & 2006 & $\begin{array}{l}\text { Narradores del siglo XX. } \\
\text { Antología de cuentistas } \\
\text { dominicanos }\end{array}$ & $\begin{array}{l}\text { Ferilibro/Letra } \\
\text { Negra Editores } \\
\text { (Guatemala) }\end{array}$ & $\begin{array}{l}\text { Diacrónica (Voces } \\
\text { recurrentes):16 Sincrónica } \\
\text { (Voces recientes): 4/7>1960 }\end{array}$ \\
\hline
\end{tabular}

Valdez divide su antología en "Voces recurrentes" (16 autores) y "Voces recientes" (7 autores) y es en este último apartado donde se encuentran cuatro autores nacidos después de 1960. Stanley propone una subdivisión en tres generaciones: generación de los sesenta (9 autores), generación de los ochenta (19 autores), de los cuales tres nacieron después de 1960 (Manuel Llibre Otero (1966), Manuel García Cartagena (1961), Nan Chevalier (1965)). Luego incorpora a cuatro autores de la generación de "la Internet", lo que también llama la generación del Nuevo Milenio: Rey Andújar (1977), Ramón Gil (1969), Johanna Díaz López (1979), Mercedes Checheen (1986). Mena no propone ninguna subdivisión, pero concede bastante importancia a los autores más recientes: 10 de los 40 autores incluidos responden al criterio manejado. Se trata de Manuel García Cartagena (1961), Ramón Tejada Holguín (1961), Aurora Arias (1962), Pastor de Moya (1965), Pedro Antonio Valdez (1968), Juan Dicent (1969), Rita Indiana Hernández (1977), Rey Emmanuel Andújar (1977), Frank Báez (1978), Mario Dávalos (1978). Como ya dijimos, Menéndez incluye a tres autores dominicanos: Rita Indiana Hernández (1977), ${ }^{7}$ Rey Andújar (1977) y Juan Dicent (1969). Puede que determinados autores no hayan sido incluidos por falta de espacio y por criterios de índole objetiva o subjetiva. Por ejemplo, la exclusión de mujeres en la antología de Valdez se debe al hecho de que quedan reservadas para un volumen por separado, al decir del antologador. Valdez no se incluye a sí mismo tampoco por ser el compilador del volumen. Con el objeto de paliar en algo este problema, también hemos tenido en cuenta si se los menciona en la introducción (indicado por (M)). Todo esto nos lleva al siguiente cuadro de autores presentes más de una vez: 


\begin{tabular}{lccccc}
\hline & $\begin{array}{c}\text { Menéndez } \\
(\mathbf{2 0 0 5})\end{array}$ & $\begin{array}{c}\text { Valdez } \\
(\mathbf{2 0 0 6})\end{array}$ & $\begin{array}{c}\text { Stanley } \\
(\mathbf{2 0 1 0})\end{array}$ & $\begin{array}{c}\text { Mena } \\
(\mathbf{2 0 1 0})\end{array}$ & Total \\
\hline Luis Martín Gómez (1961) & & $\mathrm{X}$ & $(\mathrm{M})$ & & 2 \\
Rey Andújar (1977) & $\mathrm{X}$ & $\mathrm{X}$ & $\mathrm{X}$ & $\mathrm{X}$ & 4 \\
Aurora Arias (1962) & & $(\mathrm{M})$ & $(\mathrm{M})$ & $\mathrm{X}$ & 3 \\
Manuel García Cartagena (1961) & & $\mathrm{X}$ & $\mathrm{X}$ & 2 \\
Frank Báez (1978) & & $\mathrm{X}$ & $\mathrm{X}$ & $\mathrm{X}$ & 2 \\
Manuel Llibre Otero (1966) & $\mathrm{X}$ & $(\mathrm{M})$ & & $\mathrm{X}$ & 2 \\
Juan Dicent (1969) & $\mathrm{X}$ & $(\mathrm{M})$ & & $\mathrm{X}$ & 3 \\
Rita Indiana Hernández (1977) & & $(\mathrm{M})$ & $(\mathrm{M})$ & $\mathrm{X}$ & 3 \\
Pedro Antonio Valdez (1968) & & & & & \\
\hline
\end{tabular}

Rey Andújar es el único en ser antologado por todos. Si partiéramos de la lista reducida de Menéndez, se podría constatar que Mena retoma a los dos otros, mientras que Stanley y Valdez apenas los mencionan. Con el fin de explicar estas constataciones cabe comentar el lugar de enunciación de los antologadores en relación con el 'canon crítico' y la recepción.

Los escritores Avelino Stanley y Pedro Antonio Valdez trabajaron como funcionarios bajo la dirección del Ministro-escritor, Lantigua, de 2004 a 2012. En la República Dominicana es una práctica común contratar a escritores que apoyen al partido en el poder para que ocupen puestos de funcionarios de cultura. Sobre todo Pedro Antonio Valdez (1968) parece ser el antologador 'mayor' de la República Dominicana. En 1995 ya se había dedicado a tomarle el pulso al ambiente cuentístico contemporáneo en Última flor del naufragio. Luego, fue responsable de la parte dedicada a la República Dominicana en Los nuevos caníbales. Antología de la más reciente cuentística del Caribe hispano, una edición publicada gracias al esfuerzo conjunto de tres editoriales en las tres islas de habla hispana. Para su antología de 2006 Pedro Antonio Valdez colaboró con una editorial guatemalteca Letra Negra Editores. Avelino Stanley (1959) compuso la antología a petición del entonces embajador de la República Dominicana en Colombia, Angel Lockward, como parte "de un proyecto para colocar a los autores editados en las principales librerías de América Latina" (en Stanley 11). En ambos antologadores se percibe un deseo de proyección hacia afuera desde el aparato estatal. No es de sorprender que en las descripciones biobibliográficas insistan muchas veces en que los autores seleccionados hayan sido premiados por las instancias oficiales. Stanley advierte incluso que para la última generación han sido importantes los talleres literarios: "nos ha tocado conocer sus trabajos en condición de jurado de certámenes literarios (algunos premiados, otros no)” (19). El Ministerio de Cultura fomenta a ciertos escritores e ignora a otros. Todo esto hace que las instituciones políticas, como parte del llamado 'canon crítico', influyan muchísimo en la conformación de estas antologías. Puede sorprender que no hagamos alusión a iniciativas surgidas desde el mundo académico. Resulta que en la República Dominicana casi no existen departamentos de Filología Hispánica en las Universidades y los cursos de literatura son impartidos en el marco de otras carreras. Por todo lo dicho podemos aplicar las ideas de 
Casanova: el espacio literario en la República Dominicana aún no se ha autonomizado, no se ha apartado aún de las instituciones políticas y nacionales.

Miguel D. Mena (1961), un dominicano que reside desde 1990 en Berlín, ocupa una posición bastante particular. Este ensayista, interesado en arquitectura y literatura, trabaja de consejero en la Embajada Dominicana en Alemania, pero no tiene apoyo alguno desde las instancias oficiales para sus proyectos culturales. Su sitio web "Cielonaranja" es una herramienta muy útil para informarse sobre ciertos aspectos de la vida cultural dominicana y conocer voces nuevas. En su antología diacrónica, Cuentos dominicanos. Antología de 2010, elaborada con motivo de la participación de Ediciones Cielonaranja en la Feria del Libro de Fráncfort, Mena no insiste en los premios, sólo da breves descripciones informativas de lo publicado por los distintos autores.

Ronaldo Menéndez, el antologador cubano para el Caribe hispano, es escritor y se mueve en ambientes periodísticos y de docencia en institutos de formación literaria. En su prólogo comenta brevemente los tres criterios de selección factuales: el geográfico, el temporal (nacido después de 1960), el 'literario' (un libro publicado). Dice que no hay una pretensión de definir rasgos característicos del Caribe, no existe unidad sociocultural. Habla de un "inclusivismo múltiple” (Menéndez 25). Otra observación suya importante es que los autores escogidos del Caribe hispano demuestran cierta propensión a la "ingravidez geográfica", ya que andan desperdigados entre "Estados Unidos, España y sus países exóticos" (Menéndez 26), de manera que se confirma la propuesta de la nación diluida comentada anteriormente. Los autores escogidos para la República Dominicana responden efectivamente a esta idea: son tres nómadas, que no viven en Santo Domingo, y se han desplazado bastante. De Rey Emmanuel Andújar se dice "Actualmente reside en California" (303), pero sabemos que se ha ido desplazando a menudo hasta asentarse en Chicago. No se especifica nada para Rita Indiana Hernández quien se ha ido moviendo entre Estados Unidos, Puerto Rico y la República Dominicana. Juan Dicent vive desde 2005 en Nueva York. El mismo Menéndez no comenta el porqué de la selección. Al final del volumen se encuentra una breve bio-bibliografía de los escritores incluidos, con mención de los premios nacionales obtenidos.

En relación con esto cabe preguntarse cuál es la difusión de estas antologías para que de esa manera influyan en el 'corpus' en el sentido de Pérez Cino (y a largo plazo tal vez en el 'Canon'). Resulta que las publicaciones del Ministerio y las dominicanas en general circulan poco fuera del país. La colaboración con Guatemala (en el caso de Valdez) y con Colombia (en el caso de Stanley) tal vez signifique una pequeña apertura, pero no contribuye fundamentalmente a la difusión. Stanley es consciente de este problema, ya que es en este sentido en que interpreta su título: quiere ser una Ruptura del límite. Las antologías ni siquiera son muy conocidas dentro del país, ya que no se dispone de una amplia red de distribución. La antología de bajo coste de Mena tampoco circula mucho: Cielonaranja (suspendida desde 2014) era la editorial de una sola persona. No obstante, el que se presentara el volumen en la Feria de Fráncfort además de una reedición en 2013 que se puede conseguir en Amazon, aumenta la visibilidad. Pequeñas Resistencias fue publicada en una editorial pequeña e independiente de Madrid, Páginas de Espuma, cuyo director es Juan Casamayor. No obstante, tiene distribución en las librerías más importantes de la República Dominicana y de Puerto Rico, es decir, Cuesta y La Tertulia, y se consigue 
fácilmente mediante pedidos electrónicos. Esto tiene como consecuencia que los cuentos de la literatura dominicana al alcance y susceptibles de integrarse en el 'corpus' sean a veces menos celebrados en el país.

Lo confirma otra vertiente del 'canon crítico', me refiero a la recepción en la academia y en el mundo de los críticos literarios. Stanley advierte de manera autocrítica sobre la generación del Nuevo Milenio a la que pertenece Andújar: "La crítica literaria local, sumergida en no sé qué aletargamiento, no ha hablado de ello" (18). Resulta que autores como Rita Indiana Hernández, casi ausente en los volúmenes al cuidado de antologadores del Ministerio, son efectivamente estudiados en algunos Departamentos de Letras que se ubican fuera de la República Dominicana. Es sabido que la literatura dominicana es bastante invisible en el mundo universitario en su totalidad (Rodríguez; Bustamante). No obstante, revistas como Confluencia y su editora, Esther Gimbernat-González, muy interesada en la literatura dominicana, sitios web y blogs como el de Néstor Rodríguez, profesor dominicano en la Universidad de Toronto, ayudan a dar a conocer la literatura de Quisqueya. Y después del número 'histórico' de 1988 sobre la literatura dominicana de Revista Iberoamericana, dos editores valientes decidieron en el 2011 armar un número sobre literatura dominicana contemporánea, estratégicamente combinada con la cubana, que fue publicado en septiembre del 2013. Rita Indiana Hernández, al igual que Rey Andújar, Juan Dicent (y agregaríamos a Aurora Arias), son analizados (hasta promocionados) por algunos investigadores reconocidos de universidades norteamericanas. Pienso en los dominicanos residentes fuera del país, como Fernando Valerio-Holguín (Colorado State University) o el ya mencionado Néstor Rodríguez (University of Toronto) y en los puertorriqueños Juan Duchesne Winter (University of Pittsburgh) o Julio Ramos (emérito de Berkeley). Por restringido que sea el alcance de estas iniciativas, contribuyen a difundir cierta imagen de la literatura dominicana. Interfieren en la conformación de un 'corpus' que es un tanto distinto de las propuestas para el futuro promocionadas desde dentro.

No cabe duda de que en estas evaluaciones por parte de los académicos influyen ciertas corrientes críticas y un potencial 'exportable' que rebasa lo local. Hernández, Andújar, Dicent (y Arias) reivindican una posición más autónoma y quieren obtener un capital simbólico dentro de un campo más amplio que el de su espacio nacional, proceso que no queda exento de las interferencias del mercado. Se han convertido en cierta medida en lo que Casanova llamaría autores internacionales o revolucionarios. Mena dice sobre los autores como Hernández:

[Y]a decir "lo dominicano" se hace muy cuesta arriba. La sensación de ahora es que la Isla se ha esfumado y es sólo un fondo o un trasfondo. Lo que cuenta -bien en la onda de René del Risco- es el sujeto llano, pleno, consciente de su[sic] magros límites, dispuesto a todo con tal de ser y estar finalmente ahí, adonde los deseos lo lleven, no sólo donde la Historia lo sitúe. (8)

Menéndez insiste en el experimentalismo, la vocación de universalidad y la ausencia de denuncia política, aunque sí se muestra (y no se demuestra) "el lamentable estado de las cosas" (303). Incluso Stanley apunta a lo glocal: "Sus temáticas [de la generación del Nuevo Milenio] circulan los fueros de la integración de la vida que les ha tocado, como 
la electrónica, el heavy metal, la tendencia del rap y otros elementos. No se percibe en sus obras, todavía, el interés por la denuncia social per sé como en las generaciones anteriores" (19). El adverbio "todavía" delata el presupuesto de una visión más comprometida de la literatura que ataca precisamente Menéndez. De todas formas, no se puede negar que la cultura del espectáculo tan presente en Hernández (Duchesne Winter), o la indagación en la complejidad psicológica de Andújar (De Maeseneer-Bustamante), son temas que no encontramos únicamente en estos escritores hoy en día. Se integran de manera armoniosa en el corpus internacional.

A pesar de las divergencias que existen entre lo que se propone desde dentro y desde fuera, no nos podemos deshacer de la impresión de que esta promoción en el extranjero repercute, de manera comedida, en la isla. No cambiará fundamentalmente la actitud del aparato oficial, pero postulamos que sí se van produciendo pequeńas fisuras. Un autor como Rey Andújar llega a mantener los contactos con los de dentro en un difícil equilibrio, obteniendo incluso premios y reconocimiento en el país. Así le otorgaron el Premio del Cuento Joven, Feria del Libro, en 2012. Andújar también es promocionado por escritores influyentes como el puertorriqueño Edgardo Rodríguez Juliá. Jorge Volpi lo menciona en su lista de libros recientes en El insomnio de Bolivar. La obra de Andújar va incluida en algunas versiones de la antología sobre la nueva narrativa hispanoamericana El futuro no es nuestro, proyecto lanzado por Diego Trelles Paz. Tal vez bajo la presión del éxito de Rita Indiana Hernández en el extranjero y en la academia, la escritora-cantante clausuró la Feria Internacional del Libro de Santo Domingo, no hablando de sus libros, sino cantando con su grupo de merengue-beat muy exitoso llamado Rita Indiana y los Misterios. Y en 2011, esa misma Feria Internacional la invitó a la presentación de la edición dominicana de Papi, que hasta entonces sólo contaba con una edición puertorriqueña. Muy significativamente en ese mismo año la Editorial Periférica de Cáceres sacó una edición de Papi y lanzó al mercado su novela, Nombres y animales (2013) y La mucama de Omicunlé (2015), de manera que sus novelas se van acercando un poco más al mercado español. Se podría tildar esta pequeña apertura por parte de las autoridades dominicanas como una especie de negociación, una alianza estratégica, un intento de recuperar e instrumentalizar la literatura dominicana exitosa en el extranjero. A la vez, queda mucho por hacer para que se promocione a autores de sumo interés como Aurora Arias, por ejemplo, muy marginada en su país.

De este recorrido podemos deducir que las antologías son muy importantes en las diferentes acepciones de canon. Lo que más llama la atención es que se puede observar una tensión entre academia y distribución extranjeras e instituciones políticas nacionales que inciden en el 'canon crítico' de maneras casi opuestas. En lo que se refiere al 'corpus', las antologías con distribución internacional van reforzando la presencia de determinados autores más acordes con las leyes del espacio literario mundial, de manera que se confirman las premisas de Casanova sobre la interdependencia de las esferas nacionales e internacionales. En cuanto al Canon con mayúscula, sólo el futuro nos dirá quiénes sobrevivirán en lo que sigue siendo la consagración última de cada escritor, por muy denostado que sea todo lo que se refiera al canon. 


\section{Notas}

${ }^{1}$ Este artículo ha sido escrito en el marco del proyecto de investigación "El canon en la narrativa contemporánea del Caribe hispano, de Argentina y de Chile (1990-2010)” financiado por el FWO (Research Foundation-Flanders- Belgium).

2Sería interesante estudiar el abismo entre el acercamiento propuesto en la antología compuesta por Moisés Agosto et alii, Los otros cuerpos. Antología de temática gay, lésbica y queer desde Puerto Rico y su diáspora, y la de De Camps Jiménez, Miguel, García, Mélida (comps.), Antología de la literatura gay en la República Dominicana. En “¿Existe una literatura gay en la República Dominicana?” Lam observó que hay muchos textos homofóbicos en esta antología dominicana que fue presentada en Nueva York.

${ }^{3}$ No hemos incorporado una antología diacrónica aún más reciente, hecha por José Rafael Lantigua, Ministro de Cultura hasta agosto de 2012, titulada Venir con cuentos: muestrario del cuento dominicano. Todos los autores incluidos nacieron antes de 1960. Pedro Antonio Valdez (1968) sería el único autor que podría ser tomado en consideración en nuestro análisis de este volumen, que prudentemente es llamado muestrario y no antología.

${ }^{4}$ Luis Martín Gómez (1961) y Manuel Llibre Otero (1966), también presentes en algunas antologías que estudiamos, son los únicos nacidos después de 1960, que es el criterio que manejamos. Los demás son seis autores consagrados, todos nacidos antes de 1960: Pedro Peix, Angela Hernández, Armando Almánzar Rodríguez, Marcio Veloz Maggiolo, José Alcántara Almánzar, Ligia Minaya Belliard, René del Risco Bermúdez.

5 Los volúmenes son 1. Antología del nuevo cuento español 2. Antología del cuento centroamericano contemporáneo. 3. Antología del nuevo cuento sudamericano 4.Antología del nuevo cuento norteamericano y caribeño 5. Antología del nuevo cuento español (2001-2010).

${ }^{6}$ Reproducimos el elenco. Cuba: Jorge Luis Arzola (reside en Alemania,1966), Jesús David Curbelo (1965), Alberto Garrandés (1960), Ronaldo Menéndez (reside en Espańa, 1970), Michel Perdomo García (1969), Ena Lucía Portela (1972). Puerto Rico: Elidio Torres Lagares (1965), Edgardo Nieves Mieles (1957), Pedro Cabiya (1971; reside en la República Dominicana). Cabe observar que para Nieves Mieles no se respeta el criterio de la fecha de nacimiento (posterior a 1960).

7 Puede que haya influido el que Rita Indiana Hernández ya fuera incorporada junto con Luis R. Santos (1961) a Lineas aéreas de 1999, una importante antología de la nueva narrativa hispanoamericana, compuesta por el español Eduardo Becerra. En 1999 Rita Indiana apenas había publicado algunos cuentos y poemas.

\section{Bibliografía}

Agosto, Moisés, Caleb Acevedo, David, Negrón, Luis (eds.). Los otros cuerpos. Antología de temática gay, lésbica y queer desde Puerto Rico y su diáspora. San Juan: Tiempo Nuevo, 2007. Print.

Balderston, Daniel (comp.). The Latin American Short Story. An Annoted Guide to Anthologies and Criticism. New York: Greenwood Press, 1992. Print.

Becerra, Eduardo (ed.). Lineas aéreas. Antología del cuento latinoamericano. Madrid: Lengua de Trapo, 1999. Print.

Bloom, Harold. The Western Canon. Basingstoke: Macmillan, 1995. Print.

Bobes, Marilyn, Valdez, Pedro Antonio y Gómez Berás, Carlos Roberto (eds.). Los nuevos caníbales. Antología de la más reciente cuentística del Caribe hispano. San Juan: Isla Negra, Unión y Buho, 2000. Print.

Borges, José Luis, Ocampo, Silvina, Bioy Casares, Adolfo (eds.). Antología de la literatura fantástica. Barcelona-Buenos Aires: Edhasa-Sudamericana, 1977. Print.

Bustamante Escalona, Fernanda. "Letras a ritmo desenfadado". 11 de diciembre 2013. Web. Enero, 62014. <http://60watts.cl/2013/12/columna-narrativa-dominicana-actual/>

Casanova, Pascale. La República mundial de las Letras. Barcelona: Anagrama, 2001. Print.

Colchie, Thomas (ed.). A Whistler in the Night World. Short Fiction from the Latin Americas. New York: Plume, 2002. Print.

Damrosch, David. What is World Literature? Princeton-Oxford: Princeton University Press, 2003. Print.

De Camps Jiménez, Miguel, García, Mélida (comps.). Antología de la literatura gay en la República Dominicana. Santo Domingo: Editorial Manatí, 2004. Print.

De Maeseneer, Rita. "Aprende el difícil' y las literaturas nacionales del Caribe hispano". Pasavento II.2 (verano 2014) :345-357. Print. 
---, Bustamante, Fernanda. "Los cuerpos heridos en Rita Indiana Hernández, Rey Emmanuel Andújar y Junot Díaz." Revista Iberoamericana LXXIX. 243-244 (abril-septiembre 2013): 395-414. Print.

Duchesne Winter, Juan. "Papi, la profecía, espectáculo e interrupción en Rita Indiana Hernández." Revista de critica literaria latinoamericana, XXXIV. 67 (primer semestre 2008): 289-309. Print.

Guillén, Claudio. Entre lo uno y lo diverso. Introducción a la literatura comparada. Barcelona: Crítica, 1985. Print.

Guillory, John. Cultural Capital: The Problem of Literary Canon Formation. Chicago \& London: The University of Chicago Press, 1993. Print.

Kermode, Frank. "El control institucional de la interpretación." El canon literario. Enric Sullà (ed.). Madrid: Arco/Libros, 1998: 91-112. Print.

Lam, Jimmy. “¿Existe una literatura gay en la República Dominicana?”. Web. Enero, 62014 <http://www. cielonaranja.com/jimmylanantologia.htm>.

Lantigua, José Rafael (ed.). Venir con cuentos: muestrario del cuento dominicano. Santo Domingo: Ministerio de Cultura, 2012. Print.

Manera, Danilo (ed.). Cuentos dominicanos (una antología). Madrid: Siruela, 2002. Print.

Mena, Miguel (ed.). Cuentos dominicanos. Antología. Berlín: Cielonaranja, 2010. Print.

Méndez, Danny. Narratives of Migration and Displacement in Dominican Literature. New York: Routledge, 2012. Print.

Menéndez, Ronaldo, Padilla, Ignacio, del Risco, Enrique (eds.). Pequeñas resistencias/4. Antología del nuevo cuento norteamericano y caribeño. Madrid: Páginas de Espuma, 2005. Print.

Moretti, Franco. "Conjectures on World Literature." New Left Review 1 (january-february 2000): 54-68. Print.

Pérez Cino, Waldo. "A vueltas con el canon." Iberoamericana 22 (2006): 123-42. Print.

Pozuelo Yvancos, José María y Rosa María Aradra Sánchez. Teoría del canon y literatura española. Madrid: Cátedra, 2000. Print.

Sánchez Féliz, Rubén. Viajeros del rocío. 25 narradores de la diáspora. Santo Domingo: Editora Nacional, 2008. Print.

Sánchez Prado, Ignacio (ed.). América Latina en la literatura mundial. Pittsburgh: Instituto Internacional de Literatura Iberoamericana, 2006. Print.

Stanley, Avelino (ed.). Ruptura del limite. Cuentos dominicanos. Bogotá: Cangrejo Editores, 2010. Print.

Ramos, Emelda (ed.). Antología de cuentistas dominicanas. Santo Domingo: Ministerio de Cultura de la República Dominicana, 2007. Print.

Rodríguez, Néstor. "La invisibilidad crítica de la literatura dominicana." 18 de mayo 2012. Web. Enero, 6 2014 <http://www.acento.com.do/index.php/blog/4403/78/La-invisibilidad-critica-de-la-literaturadominicana-en-Norteamerica.html>.

Torres-Saillant, Silvio. "The Tribulations of Blackness. Stages in Dominican Racial Identity." Calalloo 23.3 (2000): 1086-1111. Print.

Trelles Paz, Diego. El futuro no es nuestro. Pie de Página 2008. Web. <http://www.piedepagina.com/redux/ category/especiales/el-futuro-no-es-nuestro/>

Valdez, Pedro Antonio. Narradores del siglo XX. Antología de cuentistas dominicanos. Guatemala: FeriLibro, Letra Negra Editores, 2006. Print.

. Última Flor del Naufragio. Santo Domingo/San Juan: Ediciones Hojarasca/ Isla Negra, 1995. Print.

Volpi, Jorge. El insomnio de Bolivar. Cuatro consideraciones intempestivas sobre América Latina en el siglo XXI. Barcelona: Mondadori, 2009. Print.

Vega, Máximo. El cuento contemporáneo de Santiago (1980-2005). Santo Domingo: Ediciones Ferilibro, 2005. Print. 